\title{
Future of Theranostics: An Outlook on Precision Oncology in Nuclear Medicine
}

\author{
Thomas Langbein, Wolfgang A. Weber, and Matthias Eiber \\ Department of Nuclear Medicine, School of Medicine, Klinikum rechts der Isar, Technical University of Munich, Munich, Germany
}

\begin{abstract}
Molecular alterations in malignant disease result in the expression or upregulations of various targets that can be used for imaging and treatment with radiopharmaceuticals. This theranostic principle has acquired greater importance in personalized medicine in recent years, particularly in oncology, where advanced tumors can be treated effectively with low side effects. Since the pioneering use of ${ }^{131} \mathrm{I}$ in differentiated thyroid cancer in the 1940s, remarkable achievements in nuclear medicine endoradiotherapy have been demonstrated, mainly in the treatment of neuroendocrine neoplasms by using ${ }^{177} \mathrm{Lu}-$ labeled somatostatin analogs or in the treatment of advanced prostate cancer using prostate-specific membrane antigen-directed radionuclide therapy. Besides that, this review focuses on promising novel radiopharmaceuticals and describes their preclinical and clinical status. Radiolabeled antibodies, such as ${ }^{131}$ I-omburtamab directed against the $\mathrm{B} 7-\mathrm{H} 3$ protein on the surface of neuroblastoma cells; HuMab-5B1, a ${ }^{89} \mathrm{Zr} /{ }^{177}$ Lu-labeled antibody for the treatment of CA19-9-expressing malignancies; and ${ }^{177}$ Lu-lilotomab, a CD37 antibody for the treatment of B-cell lymphomas, are being highlighted. The neurotensin receptor ligand ${ }^{111} \mathrm{In} /{ }^{177} \mathrm{Lu}-3 \mathrm{~B}-227$ has demonstrated high potential in imaging and therapy for several malignancies (e.g., pancreatic adenocarcinomas). Targeting of the fibroblast activation protein is currently being explored for different tumor entities using PET imaging with the fibroblast activation protein inhibitor (FAPI) ${ }^{68} \mathrm{Ga}-\mathrm{FAPI}-04$, and the first therapeutic applications of 90Y-FAPI-04 have been applied. After 2 decades of rapid development in theranostics, a variety of new targets are available for further clinical investigation.
\end{abstract}

Key Words: theranostic; PSMA; neuroendocrine tumor; radionuclide therapy; neurotensin; FAPI

J Nucl Med 2019; 60:13S-19S

DOI: 10.2967/jnumed.118.220566

$\mathbf{T}$ heranostics (also called theragnostics) describes a close connection between diagnostics and consequent therapy. The term theranostic was probably first used in 1998 by John Funkhouser, who developed a test for monitoring the efficacy of a new anticoagulant drug (1). In nuclear medicine the term is increasingly being used specifically for imaging and therapy with the same radiopharmaceutical or 2 very similar radiopharmaceuticals. Of course, this concept is not new in nuclear medicine. It has, rather,

Received Jun. 4, 2019; revision accepted Jun. 24, 2019.

For correspondence or reprints contact: Wolfgang A. Weber, Department of Nuclear Medicine Klinikum rechts der Isar, Technical University Munich, Ismaninger Strasse 22, 81675 Munich, Germany.

E-mail: w.weber@tum.de

COPYRIGHT (C 2019 by the Society of Nuclear Medicine and Molecular Imaging. been used for decades for the therapy of benign and malignant thyroid diseases $(2,3)$. In recent years, however, theranostics has been successfully applied to a whole range of other malignancies, including neuroendocrine tumors (NETs) and prostate cancer in particular.

The efficacy of the therapy of NETs with radiolabeled analogs of somatostatin was ultimately proven in the NETTER-1 trial (4). This study led to the approval of ${ }^{177} \mathrm{Lu}$-DOTATATE in the United States and later in Europe (5). In parallel, ${ }^{68} \mathrm{Ga}$-labeled somatostatin analogs were approved for imaging of NETs and patient selection for peptide receptor radionuclide therapy (PRRT) $(6,7)$. More recently, the prostate-specific membrane antigen (PSMA) has been established as a theranostic target for prostate cancer. Currently, a randomized phase III registration trial is recruiting patients to prove the superiority of ${ }^{177} \mathrm{Lu}$-labeled PSMA-617 for treatment of metastatic castration-resistant prostate cancer (mCRPC) compared with the standard of care (VISION trial (8)). Various imaging agents targeting PSMA are currently in phase I-III studies, aiming for approval as novel diagnostic tools.

These 2 theranostic approaches, as well as the therapy of osseous metastases of prostate cancer with ${ }^{223} \mathrm{Ra}(4,9,10)$, have unequivocally demonstrated that radionuclide therapy can be applied not only to thyroid cancer but also to other solid tumors. Furthermore, it is now clear that radionuclide therapy not only has a palliative effect but can prolong progression-free and overall survival. Current research in theranostics is addressing new targets but-equally important - the optimization of radiopharmaceutical doses and dose schedules, investigation of combination therapies, and treatment at earlier tumor stages.

Advances in the field of preclinical oncology with a main focus on immunology hold promise to discover new molecular targets with potential future applications in theranostics. Recent developments in theranostics will definitely require adjustments in the training of nuclear medicine physicians. Compared with the current approach, which focuses on knowledge of image acquisition and interpretation as a service to our clinical partners, a strong focus is needed on clinical skills and patient management, especially in the oncologic setting. On the one hand, combined training in nuclear medicine and radiology has become increasingly important for educating imaging specialists (11). However, nuclear medicine needs to strengthen its position as a unique profession in daily, multidisciplinary clinical routine. Therefore, a clear, independent profile emphasizing specific strengths compared with neighboring disciplines (e.g., oncology, radiology, and radiation oncology) needs to be refined by, for example, updated licensing of training programs (12). In addition, nuclear medicine theranostics is currently regarded as an interesting business model by many companies. The recent acquisition of AAA and Endocyte by Novartis can be seen as an indication that economic interest in the development of 
theranostics will continue to grow (13). The approval of future radionuclide therapies will most likely generate further options, which will broaden the supply of patients with well-tolerated targeted therapies through nuclear medicine.

Because of the constantly expanding knowledge and experience gained by numerous research-active centers worldwide, it is impossible to provide a comprehensive review of new developments in theranostics. This review therefore focuses on theranostic agents that are in clinical use or have started clinical development in prospective studies. Because of space constraints, the review is also limited to solid tumors, although there are promising developments for new radioimmunotherapies of hematologic malignancies, such as CD37-targeted therapy of B-cell lymphomas (14) or CD45-targeted therapy of acute leukemias (15).

\section{CURRENT DEVELOPMENTS IN THERANOSTICS}

\section{NETs}

After the milestone achievements of the NETTER-1-trial and its major impact on the treatment of NETs using PRRT (4), numerous studies are now under way with the aim of improving molecular therapy in NETs and other somatostatin receptor-expressing tumors. In the following section, we briefly describe some of these ongoing studies.

The international phase III COMPETE study (NCT03049189) compares treatment with ${ }^{177} \mathrm{Lu}$-DOTATOC and the mammalian target of rapamycin inhibitor everolimus in patients with advanced gastroenteropancreatic NETs. Everolimus has been shown to be effective for the treatment of pancreatic and other NETs in the landmark RADIANT 3 and RADIANT 4 trials and is seen by many as the standard treatment for patients with gastroenteropancreatic NETs who progressed after treatment with somatostatin analogs (16).

In the COMPETE trial, 300 patients will be randomized to receive either $10 \mathrm{mg}$ of everolimus per day or a maximum of 4 cycles of PRRT with $7.5 \mathrm{GBq}$ of ${ }^{177} \mathrm{Lu}$-DOTATOC at 3-mo intervals. The primary endpoint of the study is progression-free survival, and the secondary endpoint is overall survival. If successful, the COMPETE trial is expected to lead to a broader use of PRRT as the primary treatment of gastroenteropancreatic NETs after failure of somatostatin analogs.

A large phase II clinical trial including more than 600 patients with any somatostatin receptor 2-positive malignancy eligible for PRRT with ${ }^{177} \mathrm{Lu}$-DOTATATE aims to investigate optimized time intervals between PRRT cycles. Progression-free survival, disease control rate, late toxicity, overall survival, and dosimetry data are being collected in a comparative, 2-armed study design examining an intensive (every $5 \mathrm{wk}$ ) versus a nonintensive (every 8-10 wk) protocol of PRRT administration (NCT03454763).

Two parallel phase II multicenter trials are recruiting patients to investigate the combination of PRRT with chemotherapy. In the first study, ${ }^{177}$ Lu-DOTATATE combined with capecitabine and temozolomide chemotherapy is compared with capecitabine and temozolomide alone in the treatment of low- to intermediate-grade pancreatic NETs. In the second study, PRRT with ${ }^{177} \mathrm{Lu}$-DOTATATE and capecitabine and temozolomide is tested versus PRRT alone in midgut NETs (NCT02358356).

DOTATATE, DOTATOC, and DOTANOC are receptor agonists that stimulate somatostatin receptors as does the natural ligand somatostatin. This stimulation leads to internalization of the receptor together with the bound ligand. Originally, it was assumed that this internalization was a prerequisite for the long intratumoral retention of radiolabeled somatostatin receptor ligands. In contrast, somatostatin receptor antagonists bind to the somatostatin receptor and block the binding of the natural ligand somatostatin but do not stimulate the receptor and do not cause internalization of the receptor (17).

Nevertheless, these antagonists have a potential advantage over agonists. Somatostatin receptor, like other $\mathrm{G}$ protein-coupled receptors, have different conformations, and agonists can bind only to so-called active conformations (17). On the cell surface, however, there are up to 100 times more somatostatin receptors in inactive conformations, and somatostatin receptor antagonists also bind to receptors that are in these inactive states (18). Ginj et al. have been the first to investigate radiolabeled somatostatin receptor antagonists for the imaging and therapy of NETs. In their experimental studies, tumor uptake of antagonists was substantially higher than that of agonists despite the lack of receptor internalization (18). High uptake and unexpectedly long retention of somatostatin receptor antagonists were also observed in small series of patients $(19,20)$.

These promising results have led to first diagnostic and therapeutic studies with the antagonists ${ }^{177} \mathrm{Lu}$-DOTA-JR11 (OPS201) (21) and ${ }^{68} \mathrm{Ga}-\mathrm{NODAGA-JR} 11$ (OPS202) (22). In these investigations, PET/CT imaging with ${ }^{68} \mathrm{Ga}-\mathrm{NODAGA}-\mathrm{JR} 11$ showed a higher image contrast and a higher sensitivity for the detection of metastases of NETs than ${ }^{68} \mathrm{Ga}$-DOTATOC (22). In a phase I therapeutic study with ${ }^{68} \mathrm{Ga} /{ }^{177} \mathrm{Lu}$-DOTA-JR11, a promising response rate of $37 \%$ was found, with a significant decrease in tumor size after only one treatment cycle (Fig. 1). However, the treatment also led to a higher hematotoxicity than observed for somatostatin receptor agonists (21). The safety and efficacy of ${ }^{177}$ Lu-DOTA-JR11 are being further investigated in ongoing multicenter phase I/II studies (NCT02592707 and NCT03773133).

\section{PSMA-Targeting Theranostics}

During the last $5 \mathrm{y}$, radioligand therapy with ${ }^{177} \mathrm{Lu}$-PSMA has rapidly evolved as a highly promising treatment for patients with mCRPC (Fig. 2). This is perhaps best demonstrated by the fact that it is already recommended by some urologic-oncologic guidelines (23), although no PSMA-targeting radiopharmaceutical has been approved by the Food and Drug Administration or the European Medicines Agency so far.

The VISION trial aims for approval of ${ }^{177} \mathrm{Lu}-\mathrm{PSMA}-617$, and enrollment is expected to end in late 2019 (8). The study randomizes 750 patients in a ${ }^{177} \mathrm{Lu}-\mathrm{PSMA}-617$ arm versus a standard-ofcare arm in a 2:1 fashion. Its primary co-endpoints are overall survival and progression-free survival. Patients who have had at least one regime of chemotherapy and secondary hormonal manipulation are eligible (NCT03511664).

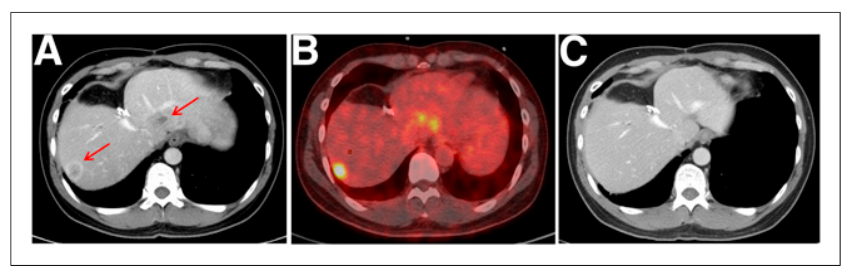

FIGURE 1. (A) CT scan of patient with gastric NET showing multiple liver metastases. (B) These metastases display high uptake of ${ }^{68} \mathrm{Ga}-$ DOTA-JR11 on PET/CT. (C) After 1 cycle of ${ }^{177}$ Lu-DOTA-JR11 (6.6 GBq), most metastases are no longer detectable on CT. 


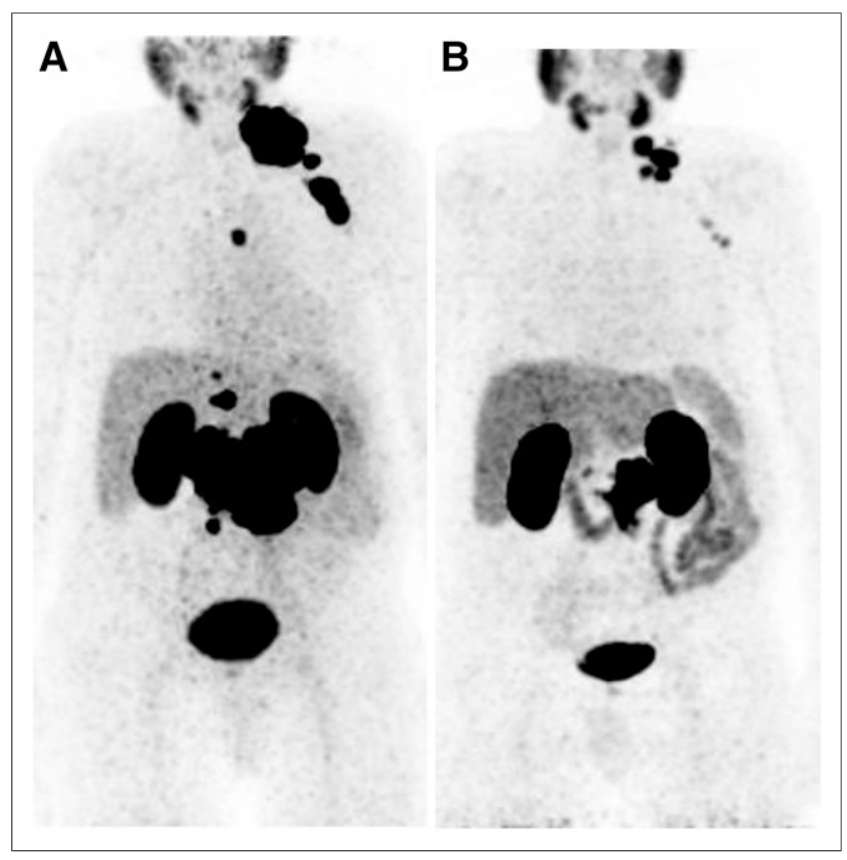

FIGURE 2. A 77-y-old patient with metastasized castration-refractory prostate cancer (initially cT3a cN1 cM0 G2b, Gleason score of 7). Patient received hormone ablation therapy, local radiation treatment, and several systemic therapies (abiraterone acetate, docetaxel, and enzalutamide). Because of progressive disease, patient was referred for 3 cycles of ${ }^{177}$ Lu-PSMA therapy. ${ }^{68}$ Ga-PSMA PET/CT showed PSMApositive nodal metastases. (A) Maximum-intensity projection. (B) Therapy response after 3 cycles of ${ }^{177}$ Lu-PSMA. (Reprinted from (59).)

The TheraP phase II trial compares ${ }^{177} \mathrm{Lu}$-PSMA-617 with the second-line chemotherapy agent cabazitaxel in mCRPC (24). Two hundred patients with metastatic prostate cancer who have progressed despite hormonal therapy and first-line chemotherapy are randomized to receive either ${ }^{177} \mathrm{Lu}-\mathrm{PSMA}$ radionuclide therapy (up to a maximum of 6 cycles of therapy) or cabazitaxel chemotherapy (up to a maximum of 10 cycles of therapy). The study will determine the effects on prostate-specific antigen (PSA) response rate (primary endpoint), pain response, progression-free survival, quality of life, and frequency and severity of adverse events (NCT03392428).

Further advances are expected when PSMA-targeted radioligand therapy is combined with other treatments. The LuPARP study investigates ${ }^{177} \mathrm{Lu}-\mathrm{PSMA}-617$ therapy and olaparib in patients with mCRPC (NCT03874884). It is a phase I, open-label, multicenter study and is designed to evaluate the safety and tolerability of olaparib in combination with ${ }^{177} \mathrm{Lu}-\mathrm{PSMA}$ in patients with $\mathrm{mCRPC}$ who have previously progressed on a novel androgen receptor-targeted agent (abiraterone or enzalutamide or apalutamide) and have not had prior exposure to platinum agents. Patients will be enrolled in 2 stages: a dose escalation phase followed by an expansion phase. The clinical and translational outcomes from this study are intended to inform the design of future phase II/III clinical trials of this combination. The PRINCE trial is a phase Ib/II study of ${ }^{177} \mathrm{Lu}-\mathrm{PSMA}-617$ therapy (up to 4 doses) in combination with pembrolizumab (up to 35 cycles) for treatment of mCRPC (NCT03658447). Primary outcome measures are adverse events and PSA response.

A different approach to increase the efficacy of PSMA-targeted radioligand therapy is the use of the $\alpha$-emitter ${ }^{225} \mathrm{Ac}$. An initial investigation has shown that targeted $\alpha$-therapy with radiolabeled
PSMA inhibitors can induce dramatic responses and that hematologic toxicity is low even in the presence of diffuse osseous and bone marrow metastases (25). However, xerostomia was significantly more severe than for ${ }^{177} \mathrm{Lu}$-labeled PSMA ligands and became the predominant dose-limiting toxicity. After an initial empiric dose-finding study, Kratochwil et al. used a fixed-dose regimen of $100 \mathrm{kBq} / \mathrm{kg}$ of body weight in a larger cohort of patients and found ${ }^{225}$ Ac-PSMA-617 to have remarkable antitumor activity (26). This study also provided preliminary evidence that responses to targeted $\alpha$-therapy can be as durable as for other therapies for castration-resistant prostate cancer.

One case series also reported on the use of ${ }^{225}$ Ac-PSMA-617 radioligand therapy in chemotherapy-naive patients with advanced metastatic prostate carcinoma. In this earlier state of prostate cancer, a PSA decline of at least $90 \%$ was observed in $82 \%$ of patients, including $41 \%$ of patients with undetectable serum PSA, who remained in remission for 12 mo after therapy (27). The remarkable therapeutic efficacy reported in this study could be achieved with reduced toxicity to the salivary glands due to deescalation of administered activities in subsequent treatment cycles. However, prospective data on the long-term efficacy and toxicity of ${ }^{225}$ Ac-PSMA-617 radioligand therapy in comparison to chemotherapy are clearly required before ${ }^{225}$ Ac-PSMA-617 radioligand therapy can be used more broadly in chemotherapy-naïve patients.

Besides PSMA-targeted radioligand therapy, PSMA-targeted radioguided surgery is an increasingly applied theranostic approach not in advanced but in early prostate cancer recurrence (Fig. 3). It uses preoperative application of $\gamma$-emitting PSMA-targeting probes that radiolabel tumor tissue (28-30). The subsequent intraoperative use of a $\gamma$-probe facilitates detection of these typically small or atypically localized lesions and improves complete resection of the recurrence. Early reports state superb rates of intraoperative tumor detection and a substantial PSA decline in a high number of patients $(29,31)$.

In a recently published series of 121 patients, a complete biochemical remission (defined as PSA $<0.2 \mathrm{ng} / \mathrm{mL}$ after PSMAtargeted radioguided surgery) was achieved in 77 patients $(65.8 \%)$ (32). The frequency was higher in patients with a preoperative PSA value below the median $(76.3 \%$ vs. $55.2 \%, P=0.018)$ and a single lesion versus multiple lesions on preoperative PSMAligand PET ( $73.7 \%$ vs. $51.2 \%, P=0.025)$. Median biochemical

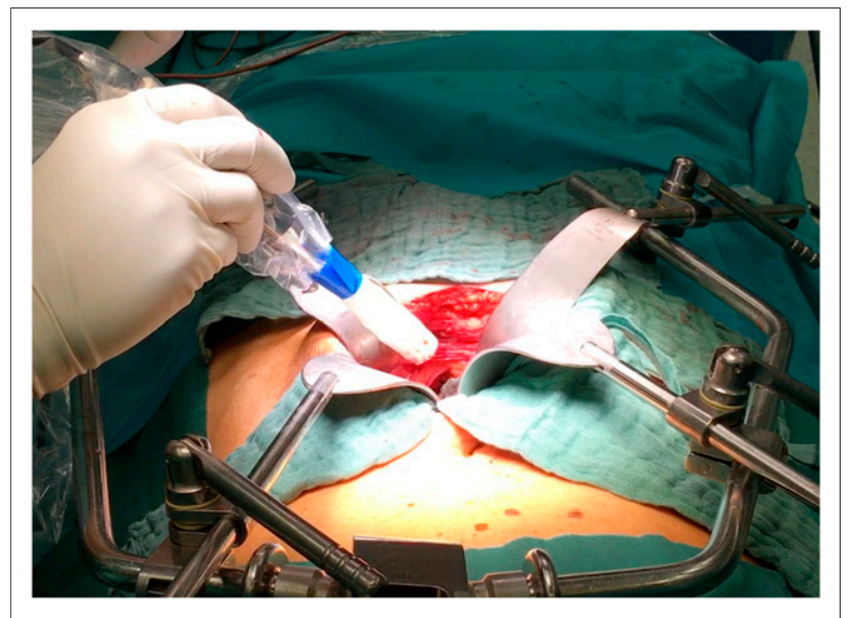

FIGURE 3. PSMA-targeted radioguided surgery. (Reprinted from (59).) 
recurrence-free survival was 6.4 mo in the whole patient cohort and 19.8 mo for patients with a complete biochemical response. A significantly longer median biochemical recurrence-free survival was observed in patients with a preoperative PSA value below the median (14.9 vs. $4.4 \mathrm{mo}, P=0.02)$ and with a single lesion on preoperative PET (14.0 vs. $2.5 \mathrm{mo}, P=0.0007)$.

\section{PERSPECTIVES ON NOVEL TARGETS AND RADIOPHARMACEUTICALS}

\section{FAP/FAPI-04}

The fibroblast activation protein (FAP) is a serine proteinase that is highly expressed on the cell surface of activated fibroblasts but not of resting fibroblasts (33). FAP is physiologically expressed in many tissues during embryonic development, but in adults it is expressed only in the context of wound healing, fibrotic processes, and the stroma of many malignancies. In histopathologic studies, FAP-positive cancer-associated fibroblasts were found in over $90 \%$ of epithelial tumors. This makes FAP a potential target for imaging and therapy of a large variety of malignancies (34).

Because of these promising characteristics, FAP antibodies, immunoconjugates, chimeric antigen receptor $\mathrm{T}$ cells, vaccines, and small-molecule FAP inhibitors (FAPIs) have already been developed (35). In 1994, a high accumulation of the ${ }^{131}$ I-labeled monoclonal antibody ${ }^{19} \mathrm{~F}$ was observed in metastases of colon carcinoma. Despite the limitations of imaging with ${ }^{131} \mathrm{I}$, metastases with a diameter of $1 \mathrm{~cm}$ were detected (36). Nevertheless, imaging with FAP antibodies was not further pursued, probably because of the success of ${ }^{18}$ F-FDG PET and the logistical challenges and high radiation exposure of imaging with radiolabeled antibodies.

However, in 2018, Haberkorn's group presented highly promising preclinical and clinical data on radiolabeled, low-molecularweight FAPIs $(35,37,38)$. These quinoline-based inhibitors were labeled with diagnostic and therapeutic radioisotopes via the chelator DOTA (35). The compound FAPI-04 showed the most favorable properties (low nanomolar affinity to FAP, almost complete internalization of radioactivity bound to FAP, and rapid blood clearance). ${ }^{68} \mathrm{Ga}$-FAPI-04 has already been used in several patients with metastatic tumors $(35,38)$. These clinical studies confirmed the favorable biodistribution expected from animal models and showed rapid tumor uptake of ${ }^{68} \mathrm{Ga}-\mathrm{FAPI}-04$ with excellent image contrast (Fig. 4A).

A first therapeutic application of ${ }^{90}$ Y-FAPI-04 has already been published (Fig. 4B) (35). High accumulation of ${ }^{90}$ Y-FAPI-04 was shown in a metastasis of a patient with advanced breast cancer. A significant improvement in symptoms was achieved with a single dose of ${ }^{90}$ Y-FAPI-04. However, FAPI-04 shows a relatively fast clearance from tumor tissue, limiting the achievable radiation dose. Hence, the effectiveness of FAP-directed radionuclide therapy can probably be improved by ligands with longer retention in the tumor tissue.

\section{Neurotensin/177Lu-3BP-227}

Neurotensin is a 13 -amino-acid neuropeptide that is physiologically found in the adult central nervous system, the gastrointestinal tract, and the myocardium $(39,40)$. Neurotensin interacts with 3 neurotensin receptors (NTSRs): NTSR1, NTSR2, and NTSR3. Neurotensin-producing neurons are widespread in the brain and involved in several neuronal functions such as pain processing, blood pressure regulation, and food intake regulation (41). Outside the brain, neurotensin acts as a hormone with numerous endocrine

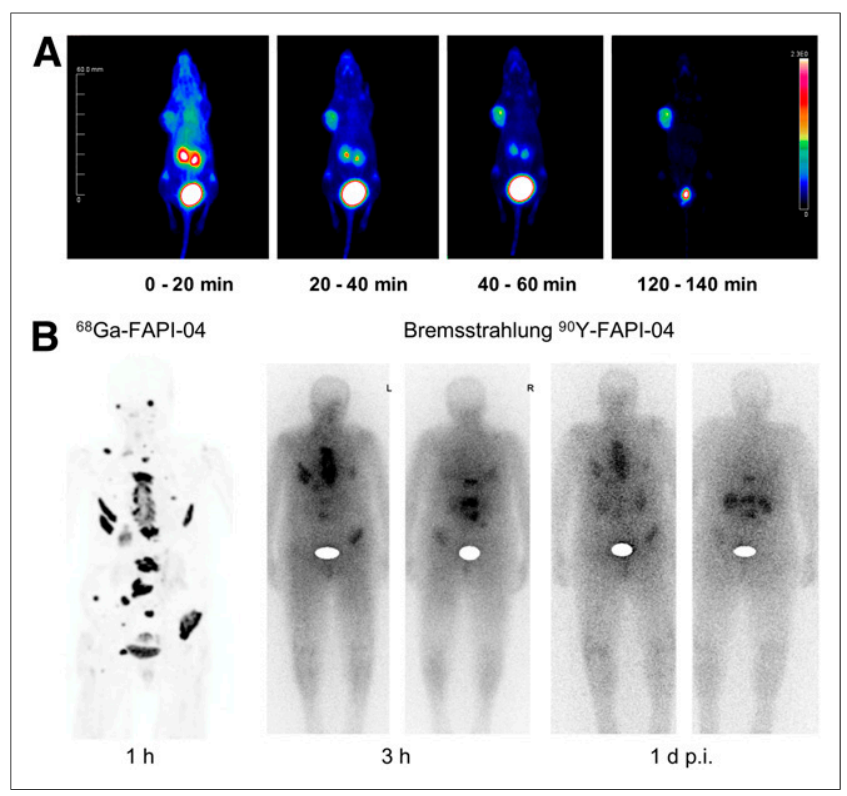

FIGURE 4. Imaging and therapy with inhibitor of FAP. (A) Biodistribution of ${ }^{68} \mathrm{Ga}-\mathrm{FAPI}-04$ in mouse tumor model expressing FAP (soft-tissue sarcoma). Rapid accumulation in tumor tissue and renal clearance without accumulation in normal tissues were observed. (Adapted from (35).) (B) ${ }^{68} \mathrm{Ga}-\mathrm{FAPI}-04$ PET (maximum-intensity projection) and planar Bremsstrahlung images of patient with osseous breast cancer metastases treated with ${ }^{90}$ Y-FAPI-04. (Reprinted from (35).)

and paracrine functions, such as regulation of intestinal motility, secretion of the pancreas, and tone of smooth vascular muscles (42).

Neurotensin and NTSRs are also expressed in various tissues during embryogenesis, and their expression is reactivated in several malignancies, such as small cell and non-small cell bronchial carcinomas, colorectal carcinomas, breast carcinomas, and pancreatic carcinomas (40). Binding of neurotensin to NTSR1 can stimulate the proliferation of tumor cells via various signaling pathways such as phospholipase C (43). In addition, the motility and invasiveness of tumor cells can be increased by activating $\rho$-kinases. For these reasons, neurotensin antagonists such as SR48692 have been developed for tumor therapy.

According to autoradiographic studies, the density of NTSR1 on pancreatic adenocarcinoma can be as high as the expression of type 2 somatostatin receptors in NETs (44). Thus, radiolabeled NTSR1 ligands represent an interesting option for radioligand therapy of pancreatic carcinomas. ${ }^{177} \mathrm{Lu}-3 \mathrm{BP}-227$ (Fig. 5A) is a highly affine nonpeptide NTSR1 antagonist (45). Preclinical studies on tumor-bearing mice have shown that ${ }^{177} \mathrm{Lu}-3 \mathrm{BP}-227 \mathrm{accu}-$ mulates in NTSR1-positive tumors with a high target-to-organ ratio compared with kidneys, liver, gastrointestinal tract, and lung (46).

On the basis of these promising data, radioligand therapy with ${ }^{177} \mathrm{Lu}-3 \mathrm{BP}-227$ was attempted in 6 patients with advanced pancreatic adenocarcinomas and no other treatment options (47). In 5 of these patients, a significant ${ }^{177} \mathrm{Lu}-3 \mathrm{BP}-227$ uptake in tumor tissue could be observed and radioligand therapy was applied. In one of the patients with extensive peritoneal carcinosis, partial remission was achieved after intraperitoneal administration of ${ }^{177} \mathrm{Lu}-3 \mathrm{BP}-$ 227 (Figs. 5A and 5B). The patient survived another 11 mo after therapy (47).

Currently, a phase I/II study is being conducted to treat advanced NTSR1-positive tumors with ${ }^{177} \mathrm{Lu}-3 \mathrm{BP}-227$ (NCT03525392). The 


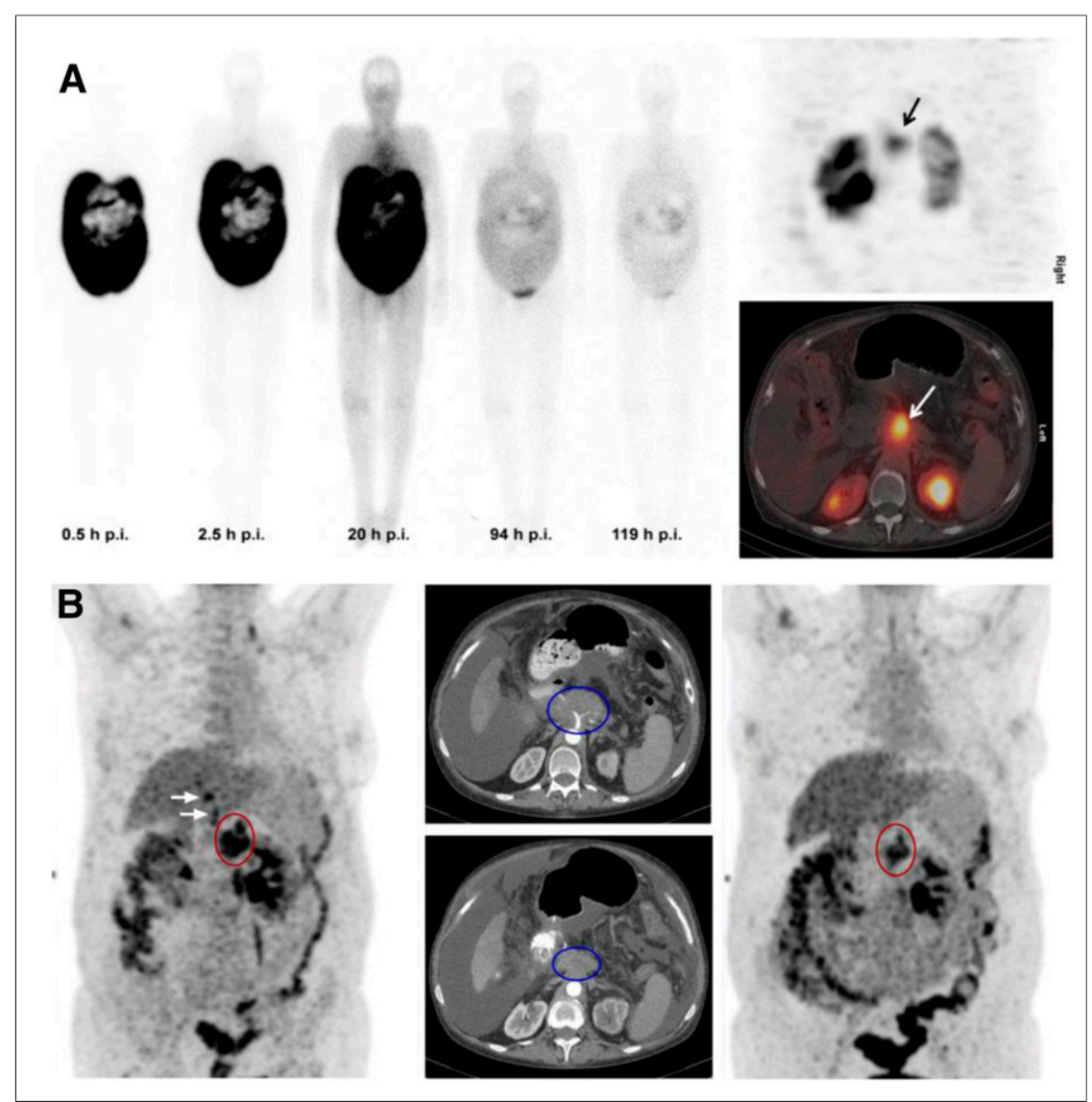

FIGURE 5. Radionuclide therapy with NTSR1 ligand. (A) Biodistribution of ${ }^{177}$ Lu-3BP-227 up to $119 \mathrm{~h}$ after intraperitoneal application in patient with pancreatic carcinoma and peritoneal carcinosis. SPECT/CT images were taken $96 \mathrm{~h}$ after injection. (B) Treatment response of patient after 3 cycles of ${ }^{177} \mathrm{Lu}-3 \mathrm{BP}-227$ on ${ }^{18} \mathrm{~F}-\mathrm{FDG}$ PET/CT imaging. Primary tumor shows decreased metabolic activity on PET (red circles) and size reduction on CT (blue oval). (Reprinted from (47).)

aim of the phase I study including 30 patients is to perform dosimetry and investigate side effects of ${ }^{177} \mathrm{Lu}-3 \mathrm{BP}-227$, as well as to determine the activity level for a phase II study. In phase II, 55 patients with pancreatic carcinoma and 70 patients with colorectal carcinoma will be treated to evaluate tumor response to ${ }^{177} \mathrm{Lu}-3 \mathrm{BP}-$ 227 therapy (NCT03525392).

\section{B7-H3 (CD276)/Omburtamab}

B7-H3 is a transmembrane B7 glycoprotein. B7 proteins play a central role in the regulation of the immune response and are important targets for the immunotherapy of malignant tumors. The interactions of B7 proteins on the surface of antigen-presenting cells with receptors from the family of CD28 receptors on the surface of $\mathrm{T}$ lymphocytes are crucial signals for T-cell activation (48). For example, the interaction of B7-1 with CD28 leads to Tcell activation, whereas the interaction of B7-1 with CTLA-4 (another protein of the family of CD28 receptors) leads to an inhibition of the immune response.

A CTLA-4 antibody (ipilimumab) used in the treatment of malignant melanomas prevents the binding of CTLA-4 to B7 and can thus induce an immune response against the tumor cells. The proteins PD-L1 and PD-L2 also belong to the B7 protein family. The interaction of these 2 proteins with the PD- 1 receptor (a CD28 receptor) on lymphocytes inhibits the immune response. PD-1 and PD-L1 antibodies (pembrolizumab, nivolumab, and atezolizumab) block this interaction and can also trigger an immune response against tumor cells. These so-called checkpoint inhibitors have been approved in recent years for the treatment of several tumor entities such as non-small cell lung cancer, melanoma, head and neck cancer, and others.

B7-H3 is overexpressed by numerous tumors (including neuroblastomas, melanomas, gliomas, and pancreatic, breast, and ovarian cancers), whereas expression in normal tissues is low (49). High expression of $\mathrm{B} 7-\mathrm{H} 3$ is associated with a poor prognosis in various malignant diseases (48). Omburtamab (8H9) is a murine $\mathrm{IgG} 1$ antibody against B7-H3 (49). After labeling with ${ }^{131} \mathrm{I}$, omburtamab was tested in patients with involvement of the central nervous system by neuroblastomas as well as for intratumoral therapy of brain stem gliomas (Fig. 6) (50-52).

In a prospective phase I/II study of 80 patients with neuroblastoma of the central nervous system, median overall survival after intrathecal therapy with ${ }^{131}$ I-omburtamab was 58 mo and thus almost 10 times longer than reported for existing therapies (external radiotherapy or surgery combined with chemotherapy). Twenty-nine percent of the patients survived for $5 \mathrm{y}$ or more. Therefore, it seems possible to cure a significant fraction of neuroblastoma patients with central nervous system involvement (52), which so far has been almost universally fatal. The rate of central nervous system side effects of intrathecal ${ }^{131}$ I-omburtamab was low, probably because of the short range of the $\beta$-radiation of ${ }^{131} \mathrm{I}$. An international phase II/III study is currently recruiting patients (NCT03275402).

\section{CA19-9/HuMab-5B1 Antibody}

CA19-9 (carbohydrate antigen 19-9 or cancer antigen 19-9 or sialyted Lewis A antigen) is a well-established tumor marker for pancreatic carcinoma (53). CA19-9 is a tetrasaccharide (54) and part of glycoproteins and glycolipids on the surface of tumor cells (53). In addition to pancreatic carcinomas, cholangiocellular, hepatocellular, gastric, colorectal, and breast carcinomas also produce CA19-9. Although CA19-9 is secreted, it is still an interesting target structure for molecular imaging and therapy, as the concentration is significantly higher in tumor tissue than in serum, and a CA19-9 ligand can therefore accumulate in the tumor tissue (55).

CA19-9HuMab-5B1 is a human IgG1 antibody that binds to C19-9 with a high affinity (dissociation constant, $0.14 \mathrm{nM}$ ) and is internalized by tumor cells. The binding is specific for C19-9, without cross-reactivity to similar saccharides such as Lewis A, Lewis X, and Lewis Y (56). In preclinical studies (Fig. 7A), an intense uptake of ${ }^{89} \mathrm{Zr}$-HuMab5B1 was seen in CA19-9-expressing tumors in mice $(>100 \%$ of the injected activity per gram of tissue), resulting in high image contrast (55). A first clinical study confirmed these promising results in 12 patients with metastatic CA19-9-expressing tumors (57). Primary tumors and metastases in 


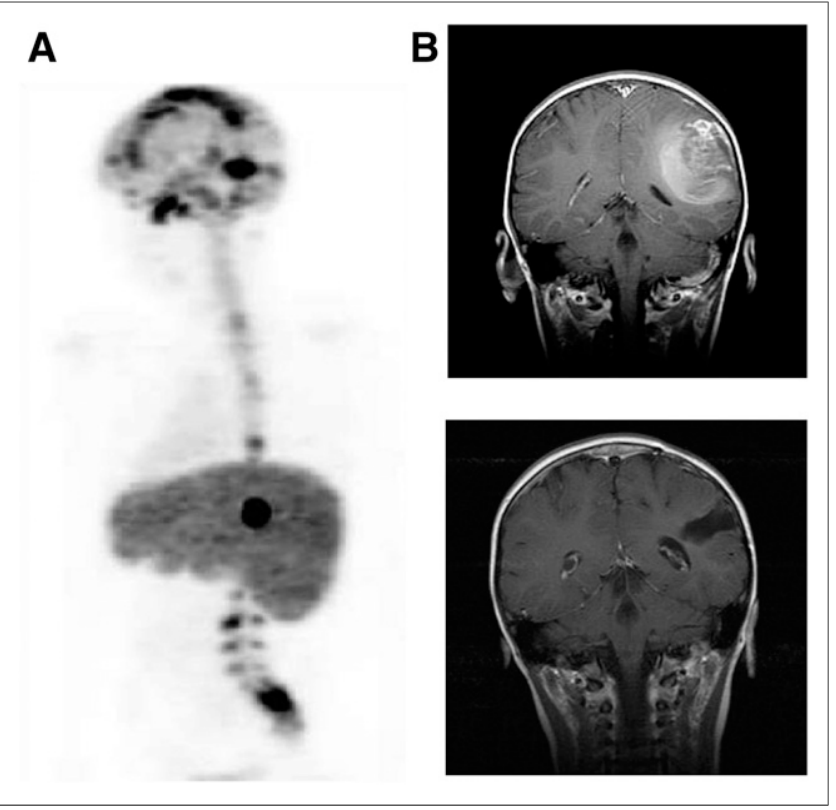

FIGURE 6. B7-H3 protein used as molecular target in treatment of neuroblastomas with cerebral metastases. (A) Distribution of ${ }^{131} \mathrm{I}$-labeled B7-H3 antibody omburtamab (8H9) $48 \mathrm{~h}$ after injection via Ommaya reservoir. (B) Coronary MRI of patient with recurrent leptomeningeal neuroblastoma before (top) and after (bottom) combined treatment with resection, external radiotherapy, and therapy with omburtamab. Patient showed no evidence of tumor progression more than 68 mo after therapy. (Reprinted with permission of (51).)

the CA19-9HuMab-5B1 PET/CT imaging showed high SUVs of up to $57 \mathrm{~g} / \mathrm{mL}$ (Fig. 7B). In addition to the metastases detected by $\mathrm{CT}$, numerous other metastases, for example, in small lymph nodes, were visualized on PET/CT.

Because of this high uptake, a phase I study on ${ }^{177} \mathrm{Lu}-\mathrm{CA} 19-$ 9HuMab-5B1 therapy was initiated and is currently recruiting patients (NCT02672917). The aim of the study is to determine

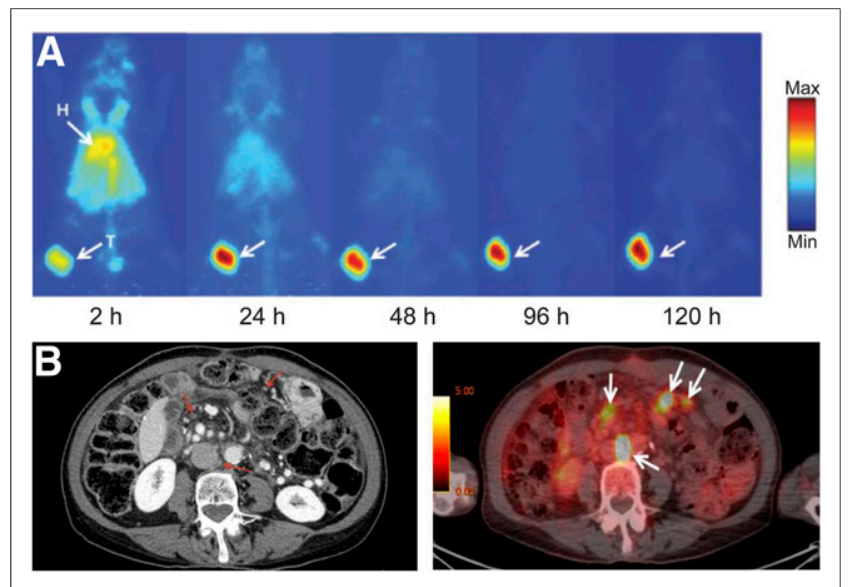

FIGURE 7. Imaging with monoclonal antibody against tetrasaccharide CA19-9. (A) Mouse imaging with CA19-9 antibody ${ }^{89}$ Zr-5B1. Increasing uptake in tumor tissue is observed up to $120 \mathrm{~h}$ after injection. (Reprinted from (55).) (B) Mesenteric lymph node metastases of patient with pancreatic carcinoma. Lymph nodes with size less than $5 \mathrm{~mm}$ on CT (red arrows) show intense uptake of ${ }^{89} \mathrm{Zr}$-5B1 $7 \mathrm{~d}$ after injection (white arrows). Color scale indicates PET images in SUV $(\mathrm{g} / \mathrm{mL})$. the maximum tolerated dose of ${ }^{177} \mathrm{Lu}-\mathrm{CA} 19-9 \mathrm{HuMab}-5 \mathrm{~B} 1$, as well as the pharmacokinetics and dosimetry of ${ }^{177} \mathrm{Lu}-\mathrm{CA} 19-9 \mathrm{HuMab}-$ 5B1 (58). Preliminary data on tumor response according to RECIST and CA19-9 changes in serum level will also be registered. The study will escalate the bone marrow dose starting from $0.5 \mathrm{~Gy}$ (58).

\section{CONCLUSION}

This review has outlined recent developments in nuclear theranostics that have already entered the clinic, as well as currently evolving therapies for new targets. Several new therapeutic approaches based on antibodies, peptidomimetics, and smallmolecule compounds have shown promising preclinical and initial clinical results. These new radiopharmaceuticals are directed against specific proteins $(\mathrm{B} 7-\mathrm{H} 3)$ or saccharides on the surface of tumor cells (CA19-9) but also against proteins produced by tumor stroma cells (FAP). Some of the radiopharmaceuticals are specific for certain tumor diseases whereas others, such as FAPI-04, are potentially suitable for a variety of tumor entities. Besides new targets, novel dose schedules and combination treatments are being studied clinically. It is therefore likely that targeted radionuclide therapy will become an ever-increasing part of clinical nuclear medicine.

\section{DISCLOSURE}

No potential conflict of interest relevant to this article was reported.

\section{REFERENCES}

1. Kelkar SS, Reineke TM. Theranostics: combining imaging and therapy. Bioconjug Chem. 2011;22:1879-1903.

2. Hertz S, Roberts A. Radioactive iodine in the study of thyroid physiology: the use of radioactive iodine therapy in hyperthyroidism. J Am Med Assoc. 1946;131: 81-86.

3. Seidlin SM, Marinelli LD, Oshry E. Radioactive iodine therapy: effect on functioning metastases of adenocarcinoma of the thyroid. J Am Med Assoc. 1946;132: $838-847$.

4. Strosberg J, El-Haddad G, Wolin E, et al. Phase 3 trial of ${ }^{177}$ Lu-dotatate for midgut neuroendocrine tumors. N Engl J Med. 2017;376:125-135.

5. Luthatera. European Medicine Agency website. https://www.ema.europa.eu/en/ medicines/human/EPAR/lutathera. Published January 17, 2018. Updated April 2, 2019. Accessed July 1, 2019.

6. NETSPOT (kit for the preparation of gallium Ga 68 dotatate injection). U.S. Food and Drug Administration website. https://www.accessdata.fda.gov/drugsatfda docs/nda/2016/208547Orig1s000TOC.cfm. Published June 21, 2016. Accessed July $1,2019$.

7. SomaKit TOC. European Medicine Agency website. https://www.ema.europa.eu/ en/medicines/human/EPAR/somakit-toc. Published February 20, 2017. Updated February 18, 2019. Accessed July 1, 2019.

8. Study of 177Lu-PSMA-617 in metastatic castrate-resistant prostate cancer (VISION). https://clinicaltrials.gov/ct2/show/NCT03511664. Published April 30, 2018. Updated June 27, 2019. Accessed July 1, 2019.

9. Parker C, Nilsson S, Heinrich D, et al. Alpha emitter radium-223 and survival in metastatic prostate cancer. N Engl J Med. 2013;369:213-223.

10. Hofman MS, Violet J, Hicks RJ, et al. $\left[{ }^{177} \mathrm{Lu}\right]-P S M A-617$ radionuclide treatment in patients with metastatic castration-resistant prostate cancer (LuPSMA trial): a single-centre, single-arm, phase 2 study. Lancet Oncol. 2018;19:825-833.

11. Larson M. Do you want an inspirational career? Choose nuclear medicine. J Nucl Med. 2019;60(suppl 2):25S-28S.

12. Czernin J, Sonni I, Razmaria A, Calais J. The future of nuclear medicine as an independent specialty. J Nucl Med. 2019;60(suppl 2):3S-12S.

13. Sherman M, Levine R. Nuclear medicine and wall street: an evolving relationship. J Nucl Med. 2019;60(suppl 2):20S-24S.

14. Blakkisrud J, Londalen A, Martinsen AC, et al. Tumor-absorbed dose for nonHodgkin lymphoma patients treated with the anti-CD37 antibody radionuclide conjugate ${ }^{177}$ Lu-lilotomab satetraxetan. J Nucl Med. 2017;58:48-54.

15. Bodet-Milin C, Kraeber-Bodere F, Eugene T, et al. Radioimmunotherapy for treatment of acute leukemia. Semin Nucl Med. 2016;46:135-146. 
16. Pavel M, O'Toole D, Costa F, et al. ENETS consensus guidelines update for the management of distant metastatic disease of intestinal, pancreatic, bronchial neuroendocrine neoplasms (NEN) and NEN of unknown primary site. Neuroendocrinology. 2016;103:172-185.

17. Bodei L, Weber WA. Somatostatin receptor imaging of neuroendocrine tumors: from agonists to antagonists. J Nucl Med. 2018;59:907-908.

18. Ginj M, Zhang H, Waser B, et al. Radiolabeled somatostatin receptor antagonists are preferable to agonists for in vivo peptide receptor targeting of tumors. Proc Natl Acad Sci USA. 2006;103:16436-16441.

19. Wild D, Fani M, Behe M, et al. First clinical evidence that imaging with somatostatin receptor antagonists is feasible. J Nucl Med. 2011;52:1412-1417.

20. Wild D, Fani M, Fischer R, et al. Comparison of somatostatin receptor agonist and antagonist for peptide receptor radionuclide therapy: a pilot study. $\mathrm{J} \mathrm{Nucl} \mathrm{Med}$. 2014;55:1248-1252.

21. Reidy DL, Pandit-Taskar N, Krebs S, et al. Theranostic trial of well differentiated neuroendocrine tumors (NETs) with somatostatin antagonists ${ }^{68} \mathrm{Ga}-\mathrm{OPS} 202$ and ${ }^{177}$ Lu-OPS201 [abstract]. J Clin Oncol. 2017;35(suppl):4094.

22. Nicolas GP, Schreiter N, Kaul F, et al. Sensitivity comparison of ${ }^{68}$ Ga-OPS202 and ${ }^{68} \mathrm{Ga}$-DOTATOC PET/CT in patients with gastroenteropancreatic neuroendocrine tumors: a prospective phase II imaging study. J Nucl Med. 2018;59:915921.

23. Guideline Program Oncology: Interdisciplinary S3-Guideline for the Early Detection, Diagnosis and Treatment of the Different Stages of Prostate Cancer [in German]. Berlin, Germany: German Cancer Society DKG, AWMF; 2018.

24. Hofman M, Emmett L, Violet JA, et al. TheraP: a randomized phase II trial of $\left[{ }^{177} \mathrm{Lu}\right]-P S M A-617$ theranostic versus cabazitaxel in progressive metastatic castration-resistant prostate cancer [abstract]. J Clin Oncol. 2019;37(suppl):TPS332.

25. Kratochwil C, Bruchertseifer F, Giesel FL, et al. ${ }^{225}$ Ac-PSMA-617 for PSMAtargeted alpha-radiation therapy of metastatic castration-resistant prostate cancer. J Nucl Med. 2016;57:1941-1944.

26. Kratochwil C, Bruchertseifer F, Rathke H, et al. Targeted alpha-therapy of metastatic castration-resistant prostate cancer with ${ }^{225}$ Ac-PSMA-617: swimmer-plot analysis suggests efficacy regarding duration of tumor control. J Nucl Med. 2018;59:795-802.

27. Sathekge M, Bruchertseifer F, Knoesen O, et al. ${ }^{225}$ Ac-PSMA-617 in chemotherapy-naive patients with advanced prostate cancer: a pilot study. Eur J Nucl Med Mol Imaging. 2019;46:129-138.

28. Maurer T, Weirich G, Schottelius M, et al. Prostate-specific membrane antigenradioguided surgery for metastatic lymph nodes in prostate cancer. Eur Urol. 2015;68:530-534.

29. Maurer T, Robu S, Schottelius M, et al. ${ }^{99 \mathrm{~m} T e c h n e t i u m-b a s e d ~ p r o s t a t e-s p e c i f i c ~}$ membrane antigen-radioguided surgery in recurrent prostate cancer. Eur Urol. 2019;75:659-666.

30. Robu S, Schottelius M, Eiber M, et al. Preclinical evaluation and first patient application of ${ }^{99 \mathrm{~m} T c-P S M A-I \& S}$ for SPECT imaging and radioguided surgery in prostate cancer. J Nucl Med. 2017;58:235-242.

31. Rauscher I, Duwel C, Wirtz M, et al. Value of ${ }^{111} \mathrm{In}$-prostate-specific membrane antigen (PSMA)-radioguided surgery for salvage lymphadenectomy in recurrent prostate cancer: correlation with histopathology and clinical follow-up. BJU Int. 2017;120:40-47.

32. Horn T, Kronke M, Rauscher I, et al. Single lesion on prostate-specific membrane antigen-ligand positron emission tomography and low prostate-specific antigen are prognostic factors for a favorable biochemical response to prostate-specific membrane antigen-targeted radioguided surgery in recurrent prostate cancer. Eur Urol. April 12, 2019 [Epub ahead of print].

33. Hamson EJ, Keane FM, Tholen S, Schilling O, Gorrell MD. Understanding fibroblast activation protein (FAP): substrates, activities, expression and targeting for cancer therapy. Proteomics Clin Appl. 2014;8:454-463.

34. Jiang GM, Xu W, Du J, et al. The application of the fibroblast activation protein alpha-targeted immunotherapy strategy. Oncotarget. 2016;7:33472-33482.

35. Lindner T, Loktev A, Altmann A, et al. Development of quinoline-based theranostic ligands for the targeting of fibroblast activation protein. $\mathrm{J} \mathrm{Nucl} \mathrm{Med}$. 2018;59:1415-1422.

36. Welt S, Divgi CR, Scott AM, et al. Antibody targeting in metastatic colon cancer: a phase I study of monoclonal antibody F19 against a cell-surface protein of reactive tumor stromal fibroblasts. J Clin Oncol. 1994;12:1193-1203.
37. Loktev A, Lindner T, Mier W, et al. A tumor-imaging method targeting cancerassociated fibroblasts. J Nucl Med. 2018;59:1423-1429.

38. Giesel FL, Kratochwil C, Lindner T, et al. FAPI-PET/CT: biodistribution and preliminary dosimetry estimate of two DOTA-containing FAP-targeting agents in patients with various cancers. J Nucl Med. 2019;60:386-392.

39. St-Gelais F, Jomphe C, Trudeau LE. The role of neurotensin in central nervous system pathophysiology: what is the evidence? J Psychiatry Neurosci. 2006;31: 229-245.

40. Osadchii OE. Emerging role of neurotensin in regulation of the cardiovascular system. Eur J Pharmacol. 2015;762:184-192.

41. Andersen JL, Schroder TJ, Christensen S, et al. Identification of the first smallmolecule ligand of the neuronal receptor sortilin and structure determination of the receptor-ligand complex. Acta Crystallogr D Biol Crystallogr. 2014;70: 451-460.

42. Qiu S, Pellino G, Fiorentino F, et al. A review of the role of neurotensin and its receptors in colorectal cancer. Gastroenterol Res Pract. 2017;2017:6456257.

43. Hou T, Shi L, Wang J, et al. Label-free cell phenotypic profiling and pathway deconvolution of neurotensin receptor-1. Pharmacol Res. 2016;108:39-45.

44. Körner M, Waser B, Strobel O, Buchler M, Reubi JC. Neurotensin receptors in pancreatic ductal carcinomas. EJNMMI Res. 2015;5:17.

45. Schulz J, Rohracker M, Stiebler M, et al. Comparative evaluation of the biodistribution profiles of a series of nonpeptidic neurotensin receptor-1 antagonists reveals a promising candidate for theranostic applications. J Nucl Med. 2016;57: 1120-1123.

46. Schulz J, Rohracker M, Stiebler M, et al. Proof of therapeutic efficacy of a ${ }^{177} \mathrm{Lu}-$ labeled neurotensin receptor 1 antagonist in a colon carcinoma xenograft model. J Nucl Med. 2017;58:936-941.

47. Baum RP, Singh A, Schuchardt C, et al. ${ }^{177} \mathrm{Lu}-3 \mathrm{BP}-227$ for neurotensin receptor 1-targeted therapy of metastatic pancreatic adenocarcinoma: first clinical results. J Nucl Med. 2018;59:809-814.

48. Janakiram M, Shah UA, Liu W, Zhao A, Schoenberg MP, Zang X. The third group of the B7-CD28 immune checkpoint family: HHLA2, TMIGD2, B7x, and B7-H3. Immunol Rev. 2017;276:26-39.

49. Modak S, Kramer K, Gultekin SH, Guo HF, Cheung NK. Monoclonal antibody $8 \mathrm{H} 9$ targets a novel cell surface antigen expressed by a wide spectrum of human solid tumors. Cancer Res. 2001;61:4048-4054.

50. Souweidane MM, Kramer K, Pandit-Taskar N, et al. Convection-enhanced delivery for diffuse intrinsic pontine glioma: a single-centre, dose-escalation, phase 1 trial. Lancet Oncol. 2018;19:1040-1050.

51. Kramer K, Kushner BH, Modak S, et al. Compartmental intrathecal radioimmunotherapy: results for treatment for metastatic CNS neuroblastoma. J Neurooncol. 2010;97:409-418.

52. Kramer K, Kushner BH, Modak S, et al. A curative approach to central nervous system metastases of neuroblastoma [abstract]. J Clin Oncol. 2017;35(suppl):10545.

53. Scarà S, Bottoni P, Scatena R. CA 19-9: biochemical and clinical aspects. $A d v$ Exp Med Biol. 2015;867:247-260.

54. Kim S, Thiessen PA, Bolton EE, et al. PubChem substance and compound databases. Nucleic Acids Res. 2016;44:D1202-D1213.

55. Viola-Villegas NT, Rice SL, Carlin S, et al. Applying PET to broaden the diagnostic utility of the clinically validated CA19.9 serum biomarker for oncology. J Nucl Med. 2013;54:1876-1882.

56. Sawada R, Sun SM, Wu X, et al. Human monoclonal antibodies to sialyl-Lewis (CA19.9) with potent CDC, ADCC, and antitumor activity. Clin Cancer Res. 2011;17:1024-1032.

57. Lohrmann C, O'Reilly E, O'Donoghue J, et al. First-in-human study of ${ }^{89} \mathrm{Zr}$ DFO-HuMab-5B1 (MVT-2163) PET/CT imaging with and without HuMab-5B1 (MVT-5873) in patients with pancreatic cancer and other CA 19-9 positive malignancies. J Nucl Med. 2017;58(suppl 1):385.

58. O'Reilly E, Lohrmann C, O'Donoghue J, et al. Phase I dose escalation study of ${ }^{177}$ Lu-HuMab-5B1 (MVT-1075) in combination with MVT-5873 as radioimmunotherapy (RIT) in subjects with relapsed/refractory pancreatic cancer or other CA19-9+ malignancies [abstract]. Cancer Res. 2018;78(13 suppl):CT140.

59. Eiber M, Fendler WP, Rowe SP, et al. Prostate-specific membrane antigen ligands for imaging and therapy. J Nucl Med. 2017;58(suppl):67S-76S. 